Published in final edited form as:

Photosynth Res. 2009 October ; 102(1): 43-51. doi:10.1007/s11120-009-9482-7.

\title{
Purification and characterization of cytochrome $c_{6}$ from Acaryochloris marina
}

\author{
Patrick D. Bell, \\ Department of Chemistry, Washington University in St. Louis, Campus Box 1137, St. Louis, MO \\ 63130-4899, USA
}

Yueyong Xin, and

Department of Chemistry, Washington University in St. Louis, Campus Box 1137, St. Louis, MO 63130-4899, USA

\section{Robert E. Blankenship \\ Department of Chemistry, Washington University in St. Louis, Campus Box 1137, St. Louis, MO 63130-4899, USA \\ Department of Biology, Washington University in St. Louis, Campus Box 1137, St. Louis, MO 63130-4899, USA}

Robert E. Blankenship: blankenship@wustl.edu

\begin{abstract}
Cytochrome $c_{6}$, (cyt $c_{6}$ ) a soluble monoheme electron transport protein, was isolated and characterized from the chlorophyll $d$-containing cyanobacterium Acaryochoris marina, the type strain MBIC11017. The protein was purified using ammonium sulfate precipitation, ion exchange and gel filtration column chromatography, and fast performance liquid chromatography. Its molecular mass and $\mathrm{pI}$ have been determined to be $8.87 \mathrm{kDa}$ and less than 4.2 , respectively, by mass spectrometry and isoelectrofocusing (IEF). The protein has an alpha helical structure as indicated by $\mathrm{CD}$ (circular dichroism) spectroscopy and a reduction midpoint potential $\left(E_{\mathrm{m}}\right)$ of $+327 \mathrm{mV}$ versus the normal hydrogen electrode (NHE) as determined by redox potentiometry. Its potential role in electron transfer processes is discussed.
\end{abstract}

\section{Keywords \\ Acaryochloris marina; Cyanobacteria; Cytochrome $c_{6}$; Photosynthesis; Chlorophyll $d$}

\section{Introduction}

Acaryochloris marina, a unicellular marine cyanobacterium, is unique among oxygenic phototrophs in the fact that it uses chlorophyll $d$ as its primary photosynthetic pigment. Compared to other chlorophyll species, chlorophyll $d$ absorbs light at longer wavelengths (Miyashita et al. 1996; Hu et al. 1998; Schenderlein et al. 2008; Swingley et al. 2005; Tomo et al. 2008). In the environments in which A. marina grows, including associations with ascidians (sea squirts) and eukaryotic macro-algae and as a part of microbial mats in the Salton Sea, a California salt lake, other chlorophyll-containing species absorb most of the shorter wavelength light. This leaves unused near infrared light from 700 to $740 \mathrm{~nm}$, which

(C) Springer Science+Business Media B.V. 2009

Correspondence to: Robert E. Blankenship, blankenship@wust l . edu. 
A. marina can absorb efficiently with its unique chlorophyll $d$ pigment (Miller et al. 2005; Bailleul et al. 2008; Cser et al. 2008; Ohasi et al. 2008; Renger and Schlodder 2008; Swingley et al. 2005; Swingley et al. 2008). The presence of this unusual pigment has made A. marina a species of interest for evolutionary analysis and possibly for bioenergy applications, and so we have begun investigating the proteins and complexes involved in its photosynthetic apparatus. One such protein is cytochrome $c_{6}$, (cyt $\left.c_{6}\right)$ which is involved in A. marina's photosynthetic electron transport chain (Bailleul et al. 2008).

Cyt $c_{6}$, a small soluble monoheme protein, is responsible for transfer of electrons between the cytochrome $b_{6} f$ complex to Photosystem I (PSI) in cyanobacterial and algal species (Bialek et al. 2008; Chida et al. 2007; Cho et al. 1999; De la Cerda et al. 1999; Inda et al. 1999; Proux-Delrourye et al. 2003; Steiner et al. 2000). The protein is categorized as a class I cytochrome with histidine and methionine serving as its fifth and sixth ligands (DiazQuintana et al. 2003; Marcaida et al. 2006; Medina et al. 1993; Worrall et al. 2007). It is a small protein, usually less than 100 amino acid residues in length and less than $10 \mathrm{kDa}$ in molecular mass (Bialek et al. 2008; Campos et al. 1993; Steiner et al. 2000). The pI of the protein can vary widely among species; typically it is acidic in algae and unicellular cyanobacteria and basic in filamentous cyanobacteria (Inda et al. 1999; Medina et al. 1993). Among cytochromes, it has a high midpoint potential $\left(E_{\mathrm{m}}\right)$ ranging between 300 and 390 $\mathrm{mV}$ versus the normal hydrogen electrode (NHE; Bialek et al. 2008; Campos et al. 1993; Inda et al. 1999; Steiner et al. 2000). The difference in $\mathrm{pI}$ and $E_{\mathrm{m}}$ among species is thought to be due to changes in the charged residues that dock with cytochrome $f$, which hints at a possible co-evolution of cyt $c_{6}$ and cytochrome $f$ (Diaz-Quintana et al. 2008; Kerfeld and Krogman 1998).

Different isoforms of cyt $c_{6}$ have been found during purification of various cyanobacterial species (Kerfeld and Krogman 1998). The genome of A. marina has been sequenced and shows two possible cyt $c_{6}$ isoforms annotated as petJ genes: AM1_2756 having 121 amino acids with a theoretical molecular weight of 10,759 Daltons for the gene product and a pI of 8.0 for the mature protein and AM1_3887 having 109 amino acids with a theoretical molecular weight of 10,819 Daltons for the gene product and a pI of 4.2 for the mature protein (Swingley et al. 2008).

In many cyanobacterial and algal species, plastocyanin (PC) a small blue copper protein, can functionally replace cyt $c_{6}$ in its electron transfer role (Bailleul et al. 2008; Chida et al. 2007; Cho et al. 1999; De la cerda 1999; Diaz-Quintana et al. 2003; Steiner et al. 2000). The sequenced A. marina genome also shows the presence of plastocyanin annotated as the petE gene: AM1_3369 (Swingley et al. 2008). When both proteins are present in a species, PC is typically the dominant protein expressed while cyt $c_{6}$ is progressively expressed under increasingly copper deficient conditions (Bialek et al. 2008; Campos et al. 1993; Cho et al. 1999; Diaz-Quintana et al. 2003; Inda et al. 1999; Worrall et al. 2007). In addition, PC and cyt $c_{6}$ are usually of similar size, pI, and $E_{\mathrm{m}}$ when both occur in the same species (Kerfeld and Krogman 1998; Medina et al. 1993). The predicted pI for the PC of A. marina is 4.34, which is very similar to the predicted value for the cyt $c_{6}$ gene product of AM1_3887. It has been suggested that the presence of these two unrelated proteins performing the same function in the same species has implications in photosynthetic evolution. When the Earth was still under anaerobic conditions, reduced iron was more soluble and readily available than reduced copper and cyt $c_{6}$ evolved first. However, when oxygen became plentiful, soluble oxidized copper was more readily available than oxidized iron and PC became the main electron transfer protein (Marcaida et al. 2006). This hypothesis is supported by the fact that only PC is present in this role in higher plants (Campos et al. 1993; Chida et al. 2007; De la Cerda et al. 1999; Diaz-Quintana et al. 2003, 2008; Inda et al. 1999; Kerfeld and Krogman 1998; Medina et al. 1993). 
In addition, other modified cyt $c_{6}$ forms have recently been discovered in higher plants and the cyanobacterium Synechococcus sp. PCC 7002 (Bialek et al. 2008; Marcaida et al. 2006; Worrall et al. 2007). The form in higher plants has been designated cytochrome $c_{6 A}$ and differs from the typical cyt $c_{6}$ by the presence of a 12 residue loop and a lower redox potential. The lower potential precludes it from serving as an effective electron transfer agent between cytochrome $b_{6} f$ and PSI and its function remains unclear (Marcaida et al. 2006; Worrall et al. 2007). Synechococcus sp. PCC 7002 has two genes petJ1 and petJ2, which code for two different isoforms of cyt $c_{6}$. The gene product of petJl is a typical cyt $c_{6}$, while that of petJ2 has a substantially lower redox potential and its function is also unclear. It has been tentatively designated as a cytochrome $c_{6 c}$ form (Bialek et al. 2008).

\section{Materials and methods}

\section{Growth conditions}

The type strain MBIC 11017 of A. marina was grown in $16 \mathrm{~L}$ of culture in carboys for two weeks in Marine BG-11 media, a modified form of Blue-Green-11 media with 3.5\% sea salt and enriched iron content equaling $0.016 \mathrm{~g} / \mathrm{L}$. Copper was present in the media as a trace metal at a concentration of $80 \mu \mathrm{g} / \mathrm{L}$. The cultures were grown aerobically with air bubbling through a sterile filter at room temperature under a light intensity of $15-17 \mu \mathrm{mol} / \mathrm{m}^{2} \mathrm{~s}$. Cells were harvested by centrifugation at 7,000 rpm. The resulting pellets weighed typically between 8 and $10 \mathrm{~g}$.

\section{Purification protocol}

The harvested cell pellets were re-suspended in $50 \mathrm{ml}$ of $0.75 \mathrm{M}$ phosphate buffer $\mathrm{pH}=7$. The high salt concentration was required to stabilize phycobillisome proteins that were being isolated simultaneously with cyt $c_{6}$. The proteinase inhibitor PMSF was added to 1 $\mathrm{mM}$ concentration to the buffer. In addition, DNAse and magnesium chloride were added to $50 \mu \mathrm{M}$ and $10 \mathrm{mM}$ concentration, respectively, to the buffer. The cells were then broken using a bead beater (Biospec Products) with silicon carbide beads. The beads were added in a 1:2 ratio to the cell solution by volume. The bead beater was run in $20 \mathrm{~s}$ intervals and then placed on ice for $5 \mathrm{~min}$ to cool. The breaking and cooling steps were repeated for a total of six times.

Following the breakage step, the cells were centrifuged at low speed $(3,850 \mathrm{rpm})$ at $15^{\circ} \mathrm{C}$ to remove beads and cellular debris. After low speed centrifugation, the resulting supernatant liquid was ultra-centrifuged at $44,000 \mathrm{rpm}$ at $15^{\circ} \mathrm{C}$ for $2 \mathrm{~h}$. The supernatant liquid from the ultra-centrifugation step was dialyzed overnight against $20 \mathrm{mM}$ HEPES buffer $\mathrm{pH}=7$ at $4^{\circ} \mathrm{C}$ to exchange buffers before proceeding with further purification.

The dialyzed solution was then brought to $60 \%$ ammonium sulfate saturation by slowing adding ammonium sulfate while stirring. After all ammonium sulfate was dissolved, the solution was left to stir at $4^{\circ} \mathrm{C}$ for $30 \mathrm{~min}$ to ensure equilibrium had been reached. The solution was centrifuged at $10,000 \mathrm{rpm}$ at $4^{\circ} \mathrm{C}$ for $15 \mathrm{~min}$. The resulting supernatant liquid, which contained the cyt $c_{6}$ fraction, was then extensively dialyzed against $20 \mathrm{mM}$ HEPES $\mathrm{pH}=7$ to remove the ammonium sulfate.

After dialysis, the solution was filtered successively with 0.45 and $0.22 \mu \mathrm{m}$ filters before being loaded onto a QSFF anion exchange column (GE Healthcare). After washing with binding buffer, the target protein was eluted with a step gradient of $0.1 \mathrm{M} \mathrm{NaCl}$ in $20 \mathrm{mM}$ HEPES buffer $\mathrm{pH}=7$ at a flow rate of $2.5 \mathrm{ml} / \mathrm{min}$. The fraction containing cyt $c_{6}$ appeared as a light pink band on the column. As the band moved near the end of the column, the eluent was monitored for cyt $c_{6}$ by measuring the UV-Vis spectra and looking for the 
characteristic cytochrome peak at $415 \mathrm{~nm}$. Collection of the eluent began once this characteristic peak appeared and ended when the UV-Vis spectra no longer showed any presence of this peak. The collected fraction totaled near $25 \mathrm{ml}$ and was stored at $4^{\circ} \mathrm{C}$.

The purification to this point was repeated over three harvests. The collected fractions from the 1st ion exchange chromatography (IEC) column were then pooled and concentrated with a 10-kDa cutoff Amicon filter to a final volume of $0.25 \mathrm{ml}$.

After concentration, the pooled fractions were loaded onto a Sephacryl-100 HR gel filtration column (GE Healthcare). The cyt $c_{6}$ fraction appeared as a pink band on the column as the sample was eluted at a flow rate of $0.5 \mathrm{ml} / \mathrm{min}$ with $20 \mathrm{mM}$ HEPES buffer $\mathrm{pH}=7$. When the pink band neared the end of the column, the eluent was collected in $3 \mathrm{ml}$ fractions. The fractions were monitored for cyt $c_{6}$ presence by measuring their UV-Vis spectra and monitoring the characteristic $415 \mathrm{~nm}$ peak. Peak fractions were collected and pooled together.

For the final purification step, the pooled fractions were loaded onto a high resolution 2nd IEC UNO Q1 column (Bio-Rad). The sample was eluted by a $1 \mathrm{ml} / \mathrm{min}$ flow rate of a linear gradient from 0-0.5 M NaCl in $20 \mathrm{mM}$ HEPES buffer controlled using a Biologic DuoFlow System (Bio-Rad). The eluent was collected in $1 \mathrm{ml}$ fractions and cyt $c_{6}$ presence was monitored by UV-Vis Spectroscopy.

\section{UV-Vis spectroscopy}

The UV-Vis spectra of the samples were taken during purification and of the final product with a Perkin Elmer Lambda 950 UV-Vis spectrophotometer and analyzed by Perkin Lambda UV WinLab Explorer software. The spectrum of the purified protein in its native reduced state was taken in $20 \mathrm{mM}$ HEPES $\mathrm{pH}=7$. The spectrum of the oxidized species was taken by adding potassium ferricyanide to $1 \mathrm{mM}$ to the purified protein sample.

\section{SDS-PAGE}

SDS-PAGE analysis was performed on the peak fraction collected from the 2nd IEC column to determine purity of the sample. The sample and Polypeptide Molecular Weight Standards (Biorad) were run on a 10-20\% acrylamide gradient precast tris-tricine SDS-PAGE gel (Biorad) designed for peptides and small proteins at $30 \mathrm{~V}$ for $1 \mathrm{~h}$ and $100 \mathrm{~V}$ for $1 \mathrm{~h}$ using a Criterion Cell (Biorad) and an EC 150 power supply (EC Apparatus Corporation).

\section{Isoelectrofocusing}

The pI of cyt $c_{6}$ was determined by isoelectrofocusing (IEF). Purified cyt $c_{6}$ sample was run on an IEF gel (Bio-Rad) with a pH gradient 3-10 with IEF standards (Bio-Rad). The gel was run at $100 \mathrm{~V}$ for $1 \mathrm{~h}$ with IEF cathode and anode buffer (Bio-Rad). The theoretical $\mathrm{pI}$ of the protein was calculated from the amino acid sequence using the compute $\mathrm{pI} / \mathrm{MW}$ tool (http:// a.expasy.org/tools/pi_tool.html.

\section{Mass spectrometry}

MALDI-TOF mass spectrometry was performed on the undigested purified cyt $c_{6}$ using a 4700 Proteomics Analyzer and Science Explorer Software (Applied Bio-systems). For the undigested protein, a sample was taken directly from the purified cyt $c_{6}$ and analyzed with the instrument to determine the mass of the intact protein. In addition, cytochrome $c$ (cyt $c$ ) from equine heart (Sigma) was used as an internal standard to calibrate the instrument to determine the experimental error for the molecular mass. 
LC-MS/MS was used to confirm the identity of the purified protein as cyt $c_{6}$ from A. marina using a reverse phase $\mathrm{C}_{18}$ column custom packed with silica media (Microohm Bioresources) and a LTQ-orbitrap mass spectrometer (Thermoscientific). The purified protein was tryptic digested following an in-solution protocol. The protein was first denatured by boiling, after which trypsin protease digestion solution (Sigma) was added to the sample. The sample was then digested overnight at $37^{\circ} \mathrm{C}$. After digestion, the sample was analyzed by the mass spectrometer following a protocol previously established in our lab (Wen et al. 2009).

The theoretical mass of the intact protein and tryptic peptides was calculated using compute pI/MW tool and FindPept tool (http://ca.expasy.org/tools/pi_tool.html; http://ca.expasy.org/ tools/findpept.html). Signal peptide prediction was performed using SignalP 3.0 Server to aid in the comparison of the experimental and theoretical masses (http://www.cbs.dtu.dk/ services/SignalP/). The MASCOT program was used to search the NCBI database for matches with the product ions from the MS/MS analysis (http://www.matrixscience.com/).

\section{Circular dichroism spectroscopy}

The circular dichroism (CD) spectrum of cyt $c_{6}$ at a concentration of $0.1 \mathrm{mg} / \mathrm{ml}$ in $10 \mathrm{mM}$ phosphate buffer $\mathrm{pH}=7$ was determined with a JASCO J-815 CD spectrometer. The raw data were converted to molar ellipiticity using Spectra Manager II software. Secondary structure analysis was performed using CD PRO Software and its analysis programs SELCON3, CDSSTR, and CONTIN (http://lamar.colostate.edu/ sreeram/CDPro/ main.html).

\section{Redox titration}

A redox titration was performed to determine the $E_{\mathrm{m}}$ of cyt $c_{6}$. Potential was controlled using a $\mathrm{CH} 620 \mathrm{C}$ potentiostat (CH Instruments) and spectral changes of cyt $c_{6}$ upon reduction and oxidation were monitored at $553 \mathrm{~nm}$ in a Perkin Elmer Lambda $950 \mathrm{UV}-\mathrm{Vis}$ spectrophotometer. The electrodes used were a platinum gauze working electrode, $\mathrm{Ag} / \mathrm{AgCl}$ reference electrode, and platinum wire auxiliary electrode. $0.1 \mathrm{M} \mathrm{NaCl}$ was added as the supporting electrolyte to the cyt $c_{6}$ sample in $20 \mathrm{mM}$ HEPES buffer $\mathrm{pH}=7$. Potassium ferricyanide, dimethyl phenylene diamine, Fe(III) EDTA, and phenazine ethosulfate were all added at $20 \mu \mathrm{M}$ to serve as redox mediators.

The fraction of cyt $c_{6}$ reduced at each potential was determined by the changes in absorbance according to Eq. 1 where $\chi$ is the fraction reduced at the respective potential, $A$ is the absorbance at $553 \mathrm{~nm}$ at the respective potential, $A_{\mathrm{o}}$ is the absorbance of the fully oxidized species at $553 \mathrm{~nm}$, and $A_{\mathrm{r}}$ is the absorbance of the fully reduced species at $553 \mathrm{~nm}$.

$$
\chi=\left(A-A_{\mathrm{o}}\right) /\left(A_{\mathrm{r}}-A_{\mathrm{o}}\right)
$$

The fraction reduced was then plotted against potential (vs. NHE) in ORIGIN and fitted to a modified Nernst equation given in Eq. 2 where $\chi$ is the fraction reduced at a given potential, $E$ is the given potential, and $E_{\mathrm{m}}$ is the midpoint potential. These equations were derived using the method developed by Dutton (1978). A best fit for the midpoint potential parameter was obtained using ORIGIN's iterative nonlinear regression analysis.

$$
\chi=10^{\left(E-E_{m}\right) / 59} /\left(10^{\left(E-E_{m}\right) / 59}+1\right)
$$




\section{Results}

\section{UV-Vis spectroscopy}

UV-Vis spectra of the purified sample in both its native reduced state and oxidized state are shown in Fig. 1. The reduced species showed the characteristic Soret band at $415 \mathrm{~nm}$, alpha band at $553 \mathrm{~nm}$, beta band at $520 \mathrm{~nm}$, and delta band at $316 \mathrm{~nm}$ along with a band at $275 \mathrm{~nm}$ representing total protein. The ratio of the absorbance at the $275 \mathrm{~nm}$ band versus at the 415 $\mathrm{nm}$ band is 0.23 . Upon oxidation, the alpha and beta bands disappear and are replaced with a broader band with a peak at $527 \mathrm{~nm}$. The Soret band decreases in intensity and blue-shifts to $409 \mathrm{~nm}$. Finally, the band at 316 disappears and a new band appears at $357 \mathrm{~nm}$.

\section{SDS-PAGE}

The SDS-PAGE of the peak fraction from the 2nd IEC column showed only one clearly distinct band between the 14 and $6.5 \mathrm{kDa}$ markers indicating a highly pure protein (Fig. 2).

\section{Isoelectric focusing}

The $\mathrm{pI}$ of cyt $c_{6}$ was determined to be less than 4.2 based on the IEF gel run with standards (Fig. 3). The predicted pI's for the two different cyt $c_{6}$ isoforms encoded by the AM1_2756 and AM1_3887 genes are 8.0 and 4.2, respectively indicating the gene product of AM1_3887 is the isoform expressed under our growth conditions.

\section{Mass spectrometry}

The MALDI-TOF spectrum of the whole protein showed peaks at $12360,8868,6180$, and $4435 \mathrm{Da}$ (Fig. 4). The set of peaks at 8,868 and 4,435 Da represent the masses of the singly charged cyt $c_{6}$ plus the mass of a proton and of the double charged cyt $c_{6}$ plus the mass of two protons, respectively, while the set of peaks at 12,360 $\mathrm{Da}$ and $6180 \mathrm{Da}$ represent the masses of the singly charged cyt $c$ plus the mass of a proton and of the double charged cyt $c$ plus the mass of twp protons, respectively. The known mass for the internal standard cytochrome $c$ from equine heart is 12,362 Da giving an experimental error in the mass accuracy of $\pm 4 \mathrm{Da}$. Therefore, the experimentally determined mass of cyt $c_{6}$ is $8,867 \pm 4$ Da.

The signal peptide analysis predicted a signal sequence cleaved between Ala 25 and Ala 26 residues. After subtracting the mass of this sequence and adding the mass of a $c$ type heme (616 Da), the theoretical mass of the protein is predicted to be 8,867 Daltons. Thus the experimental and theoretical mass of the protein are in agreement.

The predicted peptide fragments of the AM1_3887 isoform from tryptic digestion are 2276, 2673, and 2807 Da, while those from the AM1_2756 isoform are 5321, 4255, 763, 629, and $503 \mathrm{Da}$. A peak at $2276 \mathrm{Da}$ representing the tryptic digested peptide of

ADALSANGMDSADAIINQVTNGK from the AM1_3887 isoform was found with the Orbi-trap mass spectrometer which was then further analyzed by MS/MS using the ion trap part of the instrument. The product ions from the MS/MS analysis (Fig. 5) were then used in MASCOT to search for matches from the NCBI database. The first hit retrieved by MASCOT matched the A. marina cyt $c_{6}$ protein encoded by the AM1_3887 gene with a probability based mowse score of 2,096. The other hits retrieved by MASCOT were matches from other bacteria all with less significant mowse scores of lower than 100. This confirms that the purified protein is cyt $c_{6}$ from A. marina and indicates that the isoform isolated during our purification is the one coded by the AM1_3887 gene, which is also in agreement with the IEF data. 


\section{CD spectroscopy}

The CD spectrum of cyt $c_{6}$ was measured at $0.1 \mathrm{mg} / \mathrm{ml}$ concentration in $10 \mathrm{mM}$ phosphate buffer $\mathrm{pH}=7$ (Fig. 6). Two peaks appear near 209 and $222 \mathrm{~nm}$ which is characteristic of an alpha helical structure. Secondary structure analysis predicted the overall structure to be alpha helical. The average structure given by the CD PRO analysis was a $66.1 \%$ alpha helical structure with most of the remaining structure constituted from undetermined or random folds at $25.6 \%$. The analysis indicated a small amount of beta sheets (3\%) and turns (4\%) present in the sample.

\section{Redox titration}

The $E_{\mathrm{m}}$ for cyt $c_{6}$ as determined by a redox titration was $+327 \mathrm{mV}$ versus NHE. The standard error in the midpoint potential is $\pm 2 \mathrm{mV}$ obtained by the ORIGIN fitting analysis. The redox titration plot shows a reversible Nernstian behavior corresponding to a one electron oxidation/reduction reaction (Fig. 7).

\section{Discussion}

Cyt $c_{6}$ from A. marina has been purified using ammonium sulfate fractionation, column chromatography including ion exchange and gel filtration, and FPLC. Only one cyt $c_{6}$ isoform, corresponding to the AM1_3887 gene, was detected during the purification. In addition, no plastocyanin was detected during the purification. This result is in contrast with the recent analysis performed by Bailleul et al. (2008) in which they concluded that plastocyanin, not cyt $c_{6}$, was the majority soluble electron carrier between cytochrome $b_{6} f$ and PSI in A. marina. In their study, they found that after a series of actinic flashes that absorbance of A. marina whole membrane samples were photo-bleached at $554 \mathrm{~nm}$, where cyt $c$ species absorb strongly. They concluded that this bleaching could be due to the oxidation of the cytochrome $b_{6} f$ complex alone or oxidation of both the complex and cyt $c_{6}$. Since, $75 \%$ of the bleaching occurred after the first flash corresponding to one charge separation, it was concluded that the pool of electron donors to PSI associated with the bleaching was not in excess with PS I. Therefore, cyt $c_{6}$ was considered unlikely to be the soluble electron carrier because the carrier is typically in an excess of 3:1 to PSI. However, this evidence against the presence of cyt $c_{6}$ is indirect and no direct evidence for plastocyanin was presented in their analysis since its absorption spectrum is weak and broad and overlaps that of PSI (Bailleul et al. 2008).

The detection of only cyt $c_{6}$ during our purification protocol suggests that in fact the cytochrome species is a major soluble electron carrier. This conclusion would suggest along with the findings of Bailleul et al. (2008), an unusual case of an electron carrier being present in a substoichometric amount relative to PSI. However, A. marina was grown in media with a higher concentration of iron in our study than that of the Bailleul et al. (2008) study. Quantitative measurements of the levels of plastocyanin and cyt $c_{6}$ under different growth conditions would help clarify this situation.

While A. marina is unique among cyanobacteria in using chlorophyll $d$ as its primary pigment, its cyt $c_{6}$ appears to be more typical of other unicellular cyanobacteria. It is a small acidic protein with molecular mass of $8.85 \mathrm{kDa}$ and a $\mathrm{pI}$ near 4 . As indicated by $\mathrm{CD}$ Spectroscopy, it has an overall alpha helical structure with an estimated $66.1 \%$ percentage of its secondary structure being alpha helical. This percentage is slightly higher compared to those of cyt $c_{6}$ from other organisms with known crystal structures. Cyt $c_{6}$ from Acaryochloris maxima, Poryphora yeozensis, Phaeodactylum tricornutum, Cladophora glomera, and Scenedesmus obliquus have alpha helical secondary structure percentages of $59,61,57,59$, and 57\%, respectively (http://www.pdb.org/pdb/home/home.do). In addition, 
it has a high midpoint potential as indicated by redox titrations with an $E_{\mathrm{m}}$ equal to $+327 \pm 2$ $\mathrm{mV}$, which is similar to the value found in other cyanobacteria. This similarity has some bearing on the recent debate of the value of the $E_{\mathrm{m}}$ of PSI from A. marina.

Hu et al. (1998) reported the $E_{\mathrm{m}}$ for P740 in PSI in A. marina to be $+335 \mathrm{mV}$, substantially lower $(\sim 100 \mathrm{mV})$ than PSI of other cyanobacteria. The value was determined by monitoring flash-induced absorbance changes at $740 \mathrm{~nm}$ of purified PSI complexes held at different potentials by means of chemical titration. Since the PSI of A. marina absorbs light at a 0.1 eV lower quantum energy than the PSI of chlorophyll $a$ containing cyanobacteria and plants (absorption at $740 \mathrm{~nm}$ compared to $700 \mathrm{~nm}$ ), there may be a decrease in the thermodynamic driving force for the primary electron transfer steps in A. marina compared to chlorophyll $a$ containing species (Hu et al. 1998). The finding of Hu's study (1998) suggested that the decrease in $E_{\mathrm{m}}$ for the PSI of A. marina may compensate for this apparent decrease in the driving force.

However, recent studies by other research groups have determined the $E_{\mathrm{m}}$ of P740 in PSI of A. marina to be much higher ranging from +425 to $+450 \mathrm{mV}$, similar to that found in other cyanobacteria (Schelenderin et al. 2008; Bailleul et al. 2008; Tomo et al. 2008).

Schelenderin et al. 2008 found the $E_{\mathrm{m}}$ to be $+450 \mathrm{mV}$ by following flash-induced absorbance changes at $824 \mathrm{~nm}$ at varying potential by chemical titration. Bailleul et al. 2008 followed absorbance changes at $740 \mathrm{~nm}$ by both chemical and electrochemical titration and reported a value of $+425 \mathrm{mV}$. Finally, Tomo et al. (2008) determined a value of $+439 \mathrm{mV}$ by following absorbance changes at different wavelengths including $740 \mathrm{~nm}$ by electrochemical titration. Since the primary electron acceptor is not known for A. marina, it has been suggested that it could be a chlorophyll $a$ instead of $d$ and that changes in the $E_{\mathrm{m}}$ of the primary acceptor species could compensate for the difference in the energy of light absorbed (Bailleul et al. 2008; Tomo et al. 2008). Furthermore, it could be more important to maintain the high $E_{\mathrm{m}}$ for PSI to make it thermodynamically favorable for electron transfer between it and its soluble electron donor, cyt $c_{6}$ or plastocyanin (Bailleul et al. 2008; Tomo et al. 2008). While it could be possible there is a shift in the $E_{\mathrm{m}}$ of the donor as well, this is not the case found in our study where the $E_{\mathrm{m}}$ of cyt $c_{6}$ was determined to be $+327 \mathrm{mV}$, within range of the $E_{\mathrm{m}}$ of other cyanobacteria cyt $c_{6}$ (Bialek et al. 2008; Campos et al. 1993; Diaz-Quintana et al. 2008; Inda et al. 1999; Kerfeld and Krogman 1998; Steiner et al. 2000). This value would be too positive for a favorable transfer between it and PSI if the Hu et al. value is correct, but would have a similar difference in potential to PSI of other cyanobacteria, +324 $\mathrm{mV}$ (cyt $c_{6}$ ) and $+423 \mathrm{mV}$ (PSI) for Themoynechoccous elongates and $+320 \mathrm{mV}$ (cyt $c_{6}$ ) and $+450 \mathrm{mV}$ (PSI) for Synechocystis sp. PCC 6803, if the +425 to $450 \mathrm{mV}$ range for A. marina PSI is correct (Cho et al. 1999; Hou and Mauzerall 2006; Nakamura et al. 2005; ProuxDelrouyre et al. 2003).

\section{Acknowledgments}

We would like to thank the Washington University Mass Spectrometry Resource Center supported by NIH NCRR Grant No. 2P41RR000954 for their assistance. In addition, we would like to thank Jianzhong Wen for his help with the mass spectrometry and Aaron Collins for his help with the CD spectroscopy. This research was supported by a grant from the Exobiology program from NASA.

\section{Abbreviations}

$\begin{array}{ll}\text { cyt } c_{6} & \text { Cytochrome } c_{6} \\ \text { FPLC } & \text { Fast protein liquid chromatography } \\ \text { IEF } & \text { Isoelectrofocusing }\end{array}$




$\begin{array}{ll}\text { CD } & \text { Circular dichroism } \\ \boldsymbol{E}_{\mathbf{m}} & \text { Midpoint potential } \\ \text { NHE } & \text { Normal hydrogen electrode } \\ \text { A. marina } & \text { Acaryochloris marina } \\ \text { PSI } & \text { Photosystem I } \\ \text { PC } & \text { Plastocyanin } \\ \text { IEC } & \text { Ion exchange chromatography } \\ \text { MALDI-TOF } & \text { Matrix assisted laser desorption ionization-time of flight } \\ \text { LC-MS/MS } & \text { Liquid chromatography tandem mass spectrometry } \\ \chi & \text { Fraction reduced } \\ \boldsymbol{A} & \text { Absorbance } \\ \boldsymbol{A}_{\mathbf{0}} & \text { Absorbance of fully oxidized species } \\ \boldsymbol{A}_{\mathbf{r}} & \text { Absorbance of fully reduced species } \\ \boldsymbol{E} & \text { potential }\end{array}$

\section{References}

Bailleul B, Johnson X, Finazzi G, Barber J, Rappaport F, Telfer A. The thermodynamics and kinetics of electron transfer between cytochrome $b_{6} f$ and photosystem I in the chlorophyll $d$-dominated cyanobacterium, Acaryochloris marina. J Biol Chem. 2008; 283:25218-25226. [PubMed: 18635535]

Bialek W, Nelson M, Tamiola K, Kallas T, Szczepaniak A. Deeply branching $c_{6}$-like cytochromes of cyanobacteria. Biochemistry. 2008; 47:5515-5522. [PubMed: 18439023]

Campos A, Aguiar A, Hervas M, Regalla M, Navarro J, Ortega J, Xavier A, De la Rosa M, Texiera M. Cytochrome $\mathrm{c}_{6}$ from Monoraphidium braunii: a cytochrome with an unusual heme axial coordination. Eur J Biochem. 1993; 216:329-341. [PubMed: 8396033]

Chida H, Nakazawa A, Akazaki H, Hirano T, Suruga K, Ogawa M, Satoh T, Kadokura K, Yamada S, Hakamata W, Isobe K, Ito T, Ishii R, Nishio T, Sonoike K, Oku T. Expression of the algal cytochrome $c_{6}$ gene in Arabidopsis enhances photosynthesis and growth. Plant Cell Physiol. 2007; 48:948-957. [PubMed: 17548374]

Cho Y, Wang Q, Krogman D, Whitmarsh J. Extinction coefficients and midpoint potentials of cytochrome $c_{6}$ from the cyanobacteria Arthrospira maxima, Microcystis aeruginosa, and Synechocystis 6803. Biochimica et Biophysica acta. 1999; 1413:92-97. [PubMed: 10514550]

Cser K, Deak Z, Telfer A, Barber J, Vass I. Energetics of photosystem II charge recombination in Acaryochloris marina studied by thermoluminescence and flash-induced chlorophyll fluorescence measurements. Photosynth Res. 2008; 98:131-140. [PubMed: 18839331]

De la Cerda B, Diaz-Quintana A, Navarro J, Hervas M, De la Rosa M. Site-directed mutagenesis of cytochrome $c_{6}$ from Synechocystis sp. PCC 6803. The heme protein possesses a negatively charged area that may be isofunctional with the acidic patch of plastocyanin. J Biol Chem. 1999; 274:13292-13297. [PubMed: 10224089]

Diaz-Quintana A, Navarro J, Hervas M, Molina-Heredia F, De la Cerda B, De la Rosa M. A comparative structural and functional analysis of cyanobacterial plastocyanin and cytochrome $c_{6}$ as alternative electron donors to photosystem I. Photosynth Res. 2003; 75:97-110. [PubMed: 16245081]

Diaz-Quintana, A.; Hervas, M.; Navarro, J.; De la Rosa, M. Plastocyanin and cytochrome $c_{6}$ : the soluble electron carriers between the cytochrome $b_{6} f$ complex and photosystem I. In: Fromme, P., 
editor. Photosytnethic protein complexes: a structural approach. GaA, Weinheim, Germany: WileyVCH Verlag GmBH \& Co K; 2008.

Dutton P. Redox potentiometry: determination of midpoint potentials of oxidation-reduction components of biological electron-transfer systems. Methods Enzymol. 1978; 54:411-435. [PubMed: 732578]

Hou H, Mauzerall D. The A-F $\mathrm{F}_{\mathrm{X}}$ to $\mathrm{F}_{\mathrm{A} / \mathrm{B}}$ step in Synechostis 6803 photosystem I is entropy driven. JACS. 2006; 128:1580-1586.

Hu Q, Miyashita H, Iwasaki I, Kurano N, Miyachi S, Iwaki M, Itoh S. A photosystem I reaction center driven by chlorophyll $d$ in oxygenic photosynthesis. PNAS. 1998; 95:13319-13323. [PubMed: 9789086]

Inda L, Erdner D, Peleato M, Anderson D. Cytochrome $c_{6}$ isolated from the marine diatom Thalassiosira weissflogi. Phytochemistry. 1999; 51:1-4.

Kerfeld C, Krogman D. Photosynthetic cytochromes $c$ in cyanobacteria, algae, and plants. Annu Rev Plant Physiol. 1998; 49:397-425.

Marcaida M, Schlarb-Ridley B, Worrall J, Wastl J, Evans T, Bendall D, Luisi B, Howe C. Structure of cytochrome $c_{6 A}$, a novel dithio-cytochrome of Arabidopsis thaliana, and its reactivity with plastocyanin: implications for function. J Mol Biol. 2006; 360:968-977. [PubMed: 16815443]

Medina M, Diaz A, Hervaz M, Navarro J, Gomez-Marino C, de la rosa M, Tollin G. A comparative laser-flash absorption spectroscopy study of Anabaena PCC 7119 plastocyanin and cytochrome $c_{6}$ photooxidation by photosystem I particles. Eur J Biochem. 1993; 213:1133-1138. [PubMed: 8504808]

Miller S, Augustine S, Olson T, Blankenship R, Selker J, Wood A. Discovery of a free-living chlorophyll $d$ - producing cyanobacterium with a hybrid proteobacterial/cyanobacterial subunit rRNA gene. PNAS. 2005; 102:850-855. [PubMed: 15637160]

Miyashita H, Ikemoto H, Kurano N, Adachi K, Chihara M, Miyachi S. Chlorophyll $d$ as a major pigment. Nature. 1996; 383:402.

Nakamura A, Suzawa T, Kato Y, Watanbe T. Significant species dependence of P700 redox potential as verified by spectroelectrochemistry: comparison of spinach and Thermosynnechoccus elongatus. FEBS Lett. 2005; 579:2273-2276. [PubMed: 15848157]

Ohashi S, Miyashita H, Okada N, Iemura T, Watanabe T, Kobayashi M. Unique photosystems in Acaryochloris marina. Photosynth Res. 2008; 98:141-149. [PubMed: 18985431]

Proux-Delrourye V, Demaille C, Leibl W, Setif P, Bottin H, Bourdillon C. Electrocatalytic investigation of lightinduced electron transfer between cytochrome $c_{6}$ and photosystem I. JACS. 2003; 125:13686-13692.

Renger T, Schlodder E. The primary electron donor of photosystem II of the cyanobacterium Acaryochloris marina is a chlorophyll $d$ and the water oxidation is driven by a chlorophyll $a$ / chlorophyll $d$ heterodimer. J Phys Chem B. 2008; 112:7351-7354. [PubMed: 18512893]

Schlenderin, M.; Cetin, M.; Schlodder, E.; Benson, S.; Sharma, P.; Barber, J.; Telfer, A. Reassessment of the redox potential of P740: the primary electron donor in photosystem I of the chlorophyll $d$ containing cyanobacterium Acaryochloris marina. In: Allen, J.; Gantt, E.; Golbeck, J.; Osmond, B., et al., editors. Photosynthesis: energy from the Sun: 14th International Congress on Photosynthesis; Springer, New York. 2008.

Steiner J, Serrano A, Allmaeir G, Jakowitsch J, Loffelhardt W. Cytochrome $c_{6}$ from Cyanophora paradoxa Characterization of the protein and the cDNA of the precursor and import into isolated cyanelles. Eur J Biochem. 2000; 267:4232-4241. [PubMed: 10866828]

Swingley W, Hohmann-Marriot M, Olsen T, Blankenship R. Effects of iron on growth and ultrastructure of Acaryochloris marina. Appl Environ Microbiol. 2005; 71:8606-8610. [PubMed: 16332853]

Swingley W, Chen M, Cheung C, Conrad A, Dejesa L, Hao J, Honchak B, Karbach L, Kurdoglu A, Lahiri S, Mastrian D, Miyashita H, Page L, Ramakrishna P, Satoh S, Sattley W, Shimada Y, Taylor H, Tomo T, Tsuchiya T, Wang Z, Raymond J, Mimuro M, Blankenship R. Niche adaptation and genome expansion in the chlorophyll $d$-producing cyanobacterium Acaryochloris marina. PNAS. 2008; 105:2005-2010. [PubMed: 18252824] 
Tomo T, Kato Y, Suzuki T, Akimoto S, Okubo T, Noguchi T, Hasewaga K, Tohru T, Tanaka K, Michitaka Fukuya, Naoshi Dohmae, Watanbe T, Mimuro M. Characterization of highly purified photosystem I complexes from the chlorophyll $d$-dominated cyanobacterium Acaryochloris MBIC 11017. JBC. 2008; 283:18198-18209.

Wen J, Zhang H, Gross M, Blankenship R. Membrane orientation of the FMO antenna protein from Chlorobaculum tepidum as determined by mass spectrometry-based footprinting. Proc Natl Acad Sci USA. 2009

Worrall J, Schlarb-Ridley B, Reda T, Maracaida M, Moorlen R, Wastl J, Hirst J, Bendall D, Luisi B, Howe C. Modulation of heme redox potential in the cytochrome $c_{6}$ family. JACS. 2007; 129:9468-9475. 


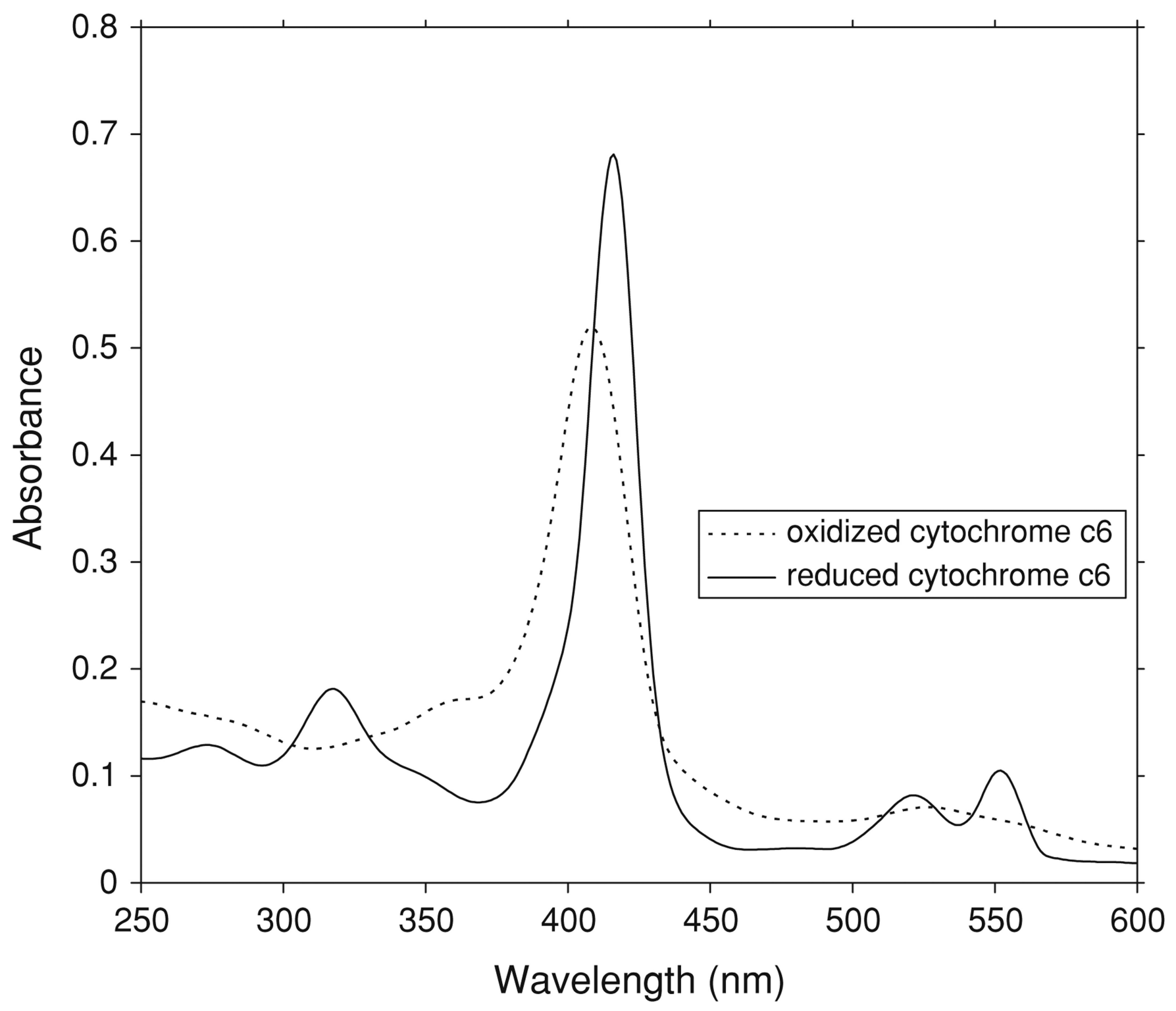

Fig. 1.

UV-Vis Spectra of purified cyt $c_{6}$ from $A$. marina with the solid line representing the reduced form and the dashed line representing the oxidized form 


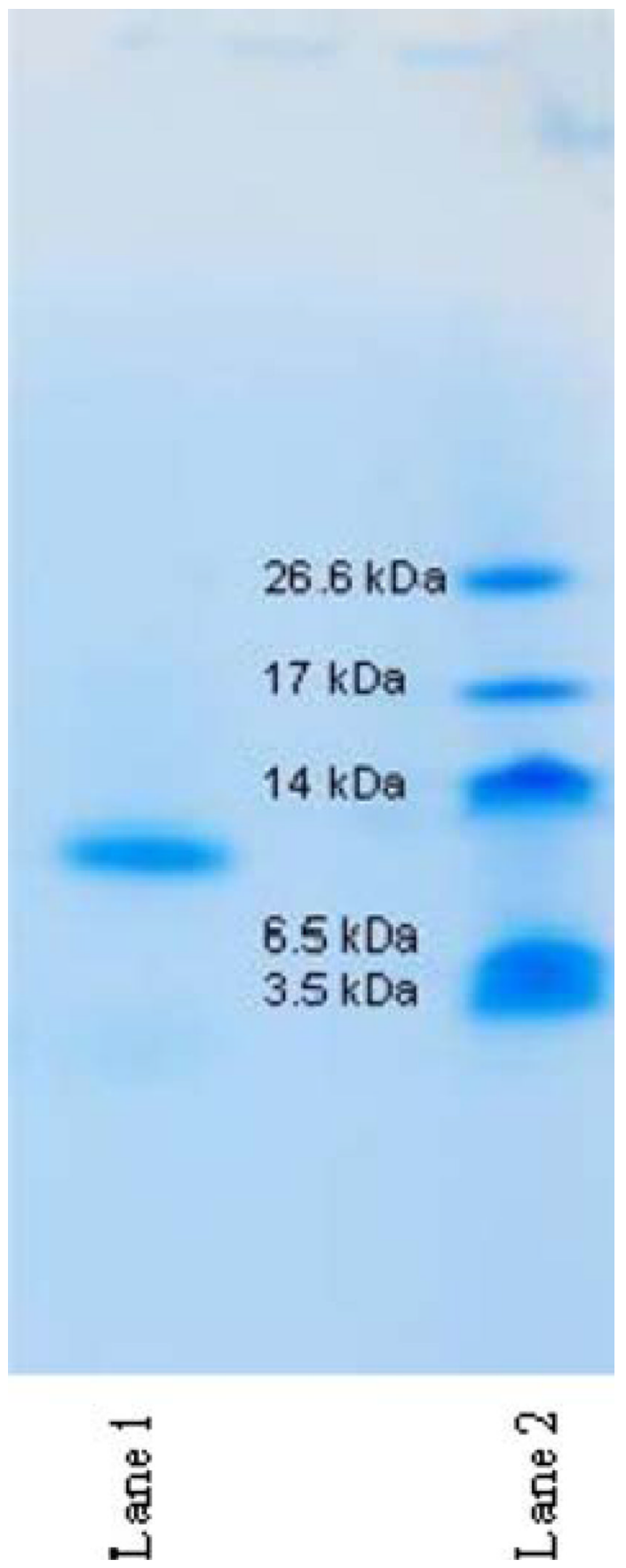

Fig. 2.

10-20\% Gradient SDS-PAGE Gel of cyt $c_{6}$ from A. marina. Lane 1 cyt $c_{6}$ sample from 2 nd IEC; Lane 2 Polypeptide Molecular Weight Standards 

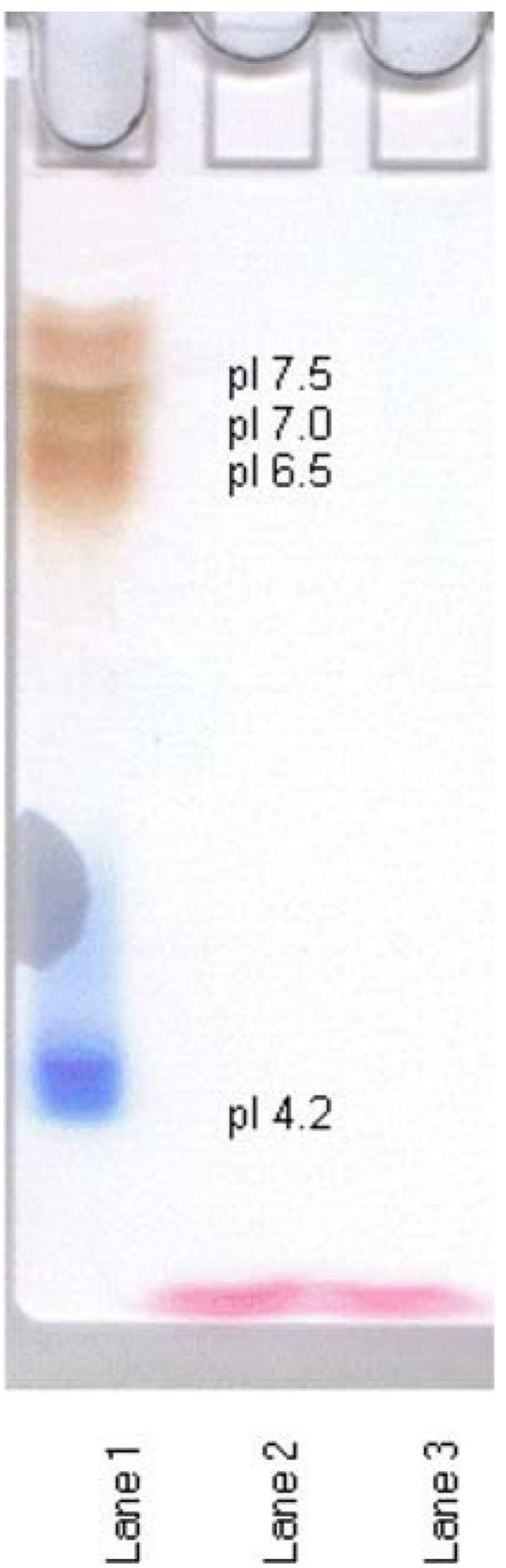

Fig. 3.

IEF Gel of purified cyt $c_{6}$ from A. marina. Lanel IEF Standards; Lanes 2 and 3 purified cyt $c_{6}$ 


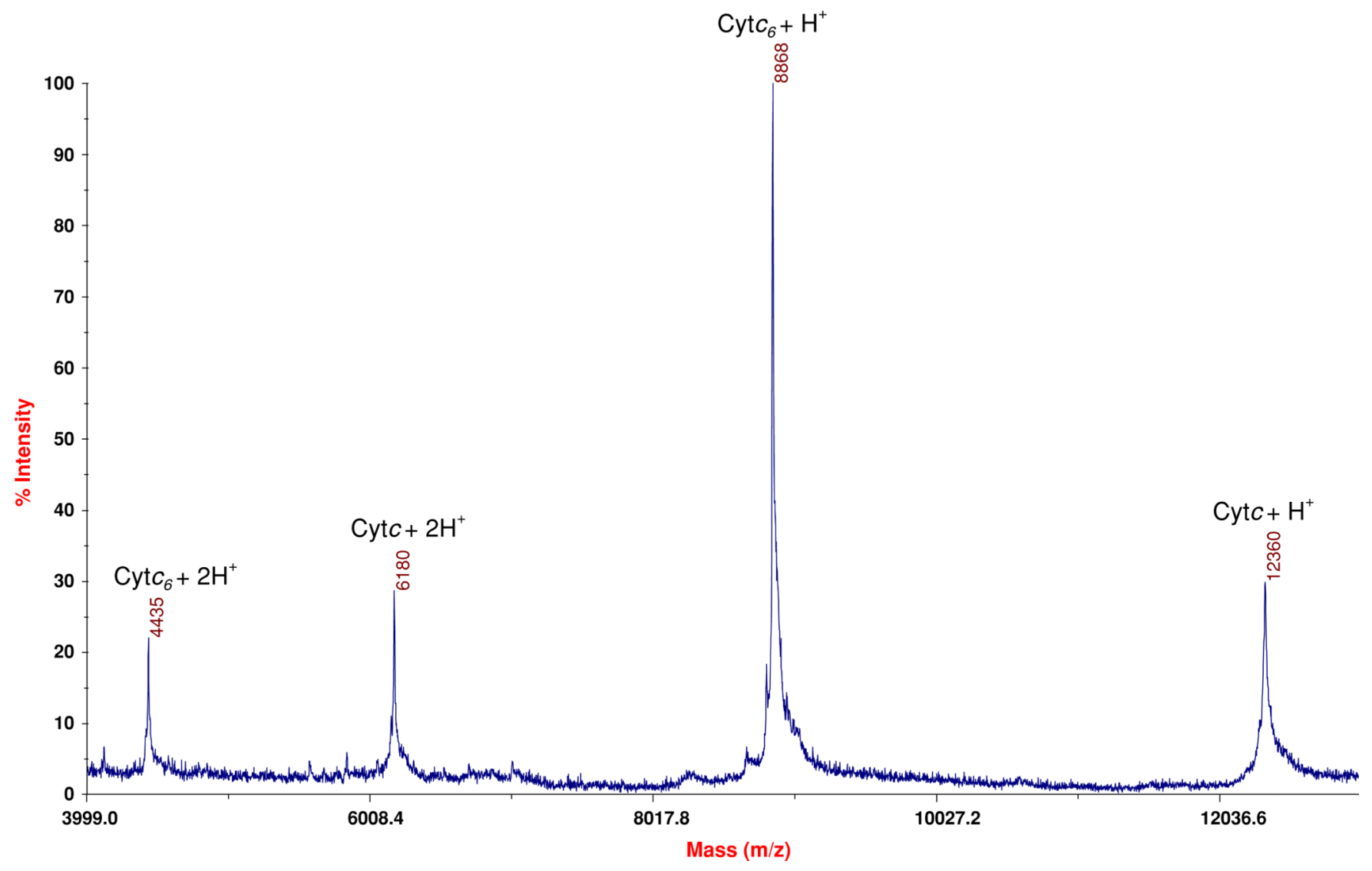

Fig. 4.

MALDI-TOF Spectrum of isolated whole mass cyt $c_{6}$ from $A$. marina with cyt $c$ from equine heart as an internal standard 
(a) $\begin{array}{lllllllllll}\mathrm{y}_{21} & \mathrm{y}_{19} & \mathrm{y}_{17} & \mathrm{y}_{15} & \mathrm{y}_{13} & \mathrm{y}_{11} & \mathrm{y}_{9} & \mathrm{y}_{7} & \mathrm{y}_{5} & \mathrm{y}_{3} & \mathrm{y}_{1}\end{array}$

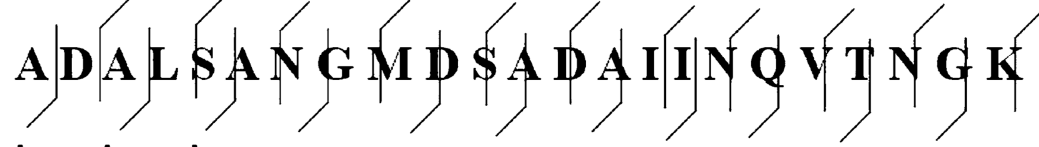

$\begin{array}{lllllllllll}b_{1} & b_{3} & b_{5} & b_{7} & b_{9} & b_{11} & b_{13} & b_{15} & b_{17} & b_{19} & b_{21}\end{array}$

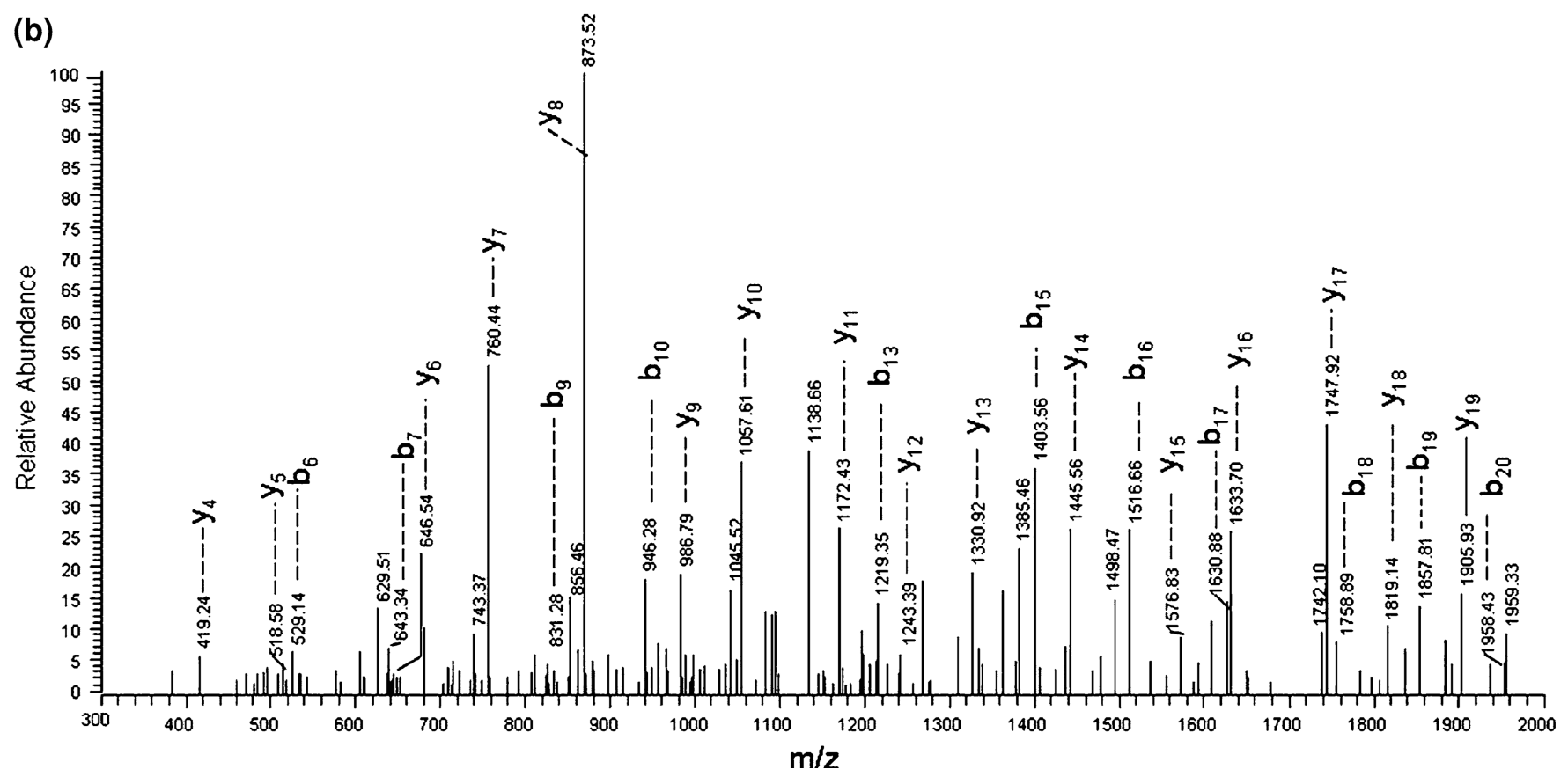

Fig. 5.

LC-MS/MS Product ions from (a) Tryptic digested peptide

ADALSANGMDSADAIINQVTNGK and (b) and their ms/ms Mass spectra from purified cyt $c_{6}$ from $A$. marina 


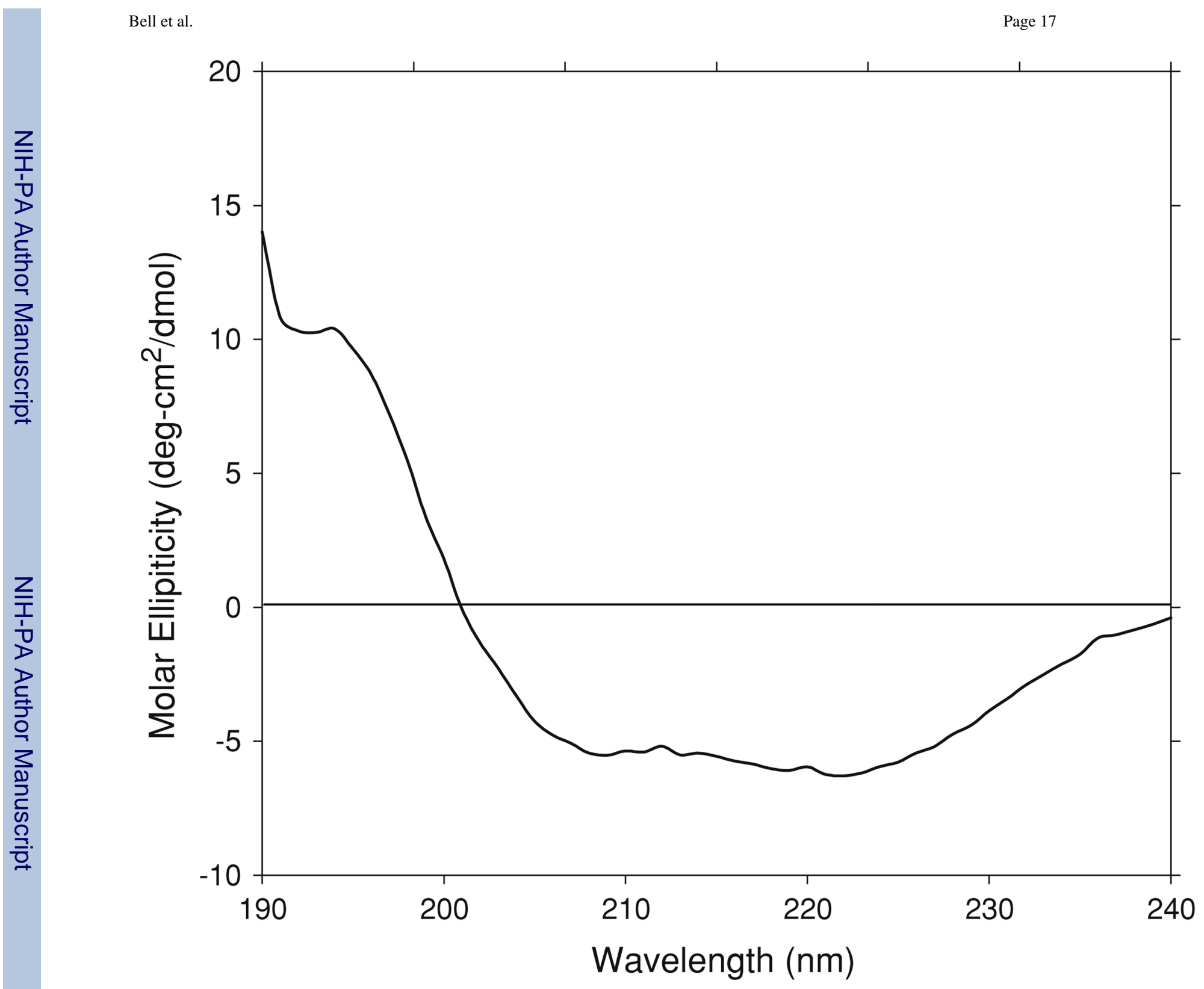

Fig. 6.

CD spectrum of purified cyt $c_{6}$ from A. marina 


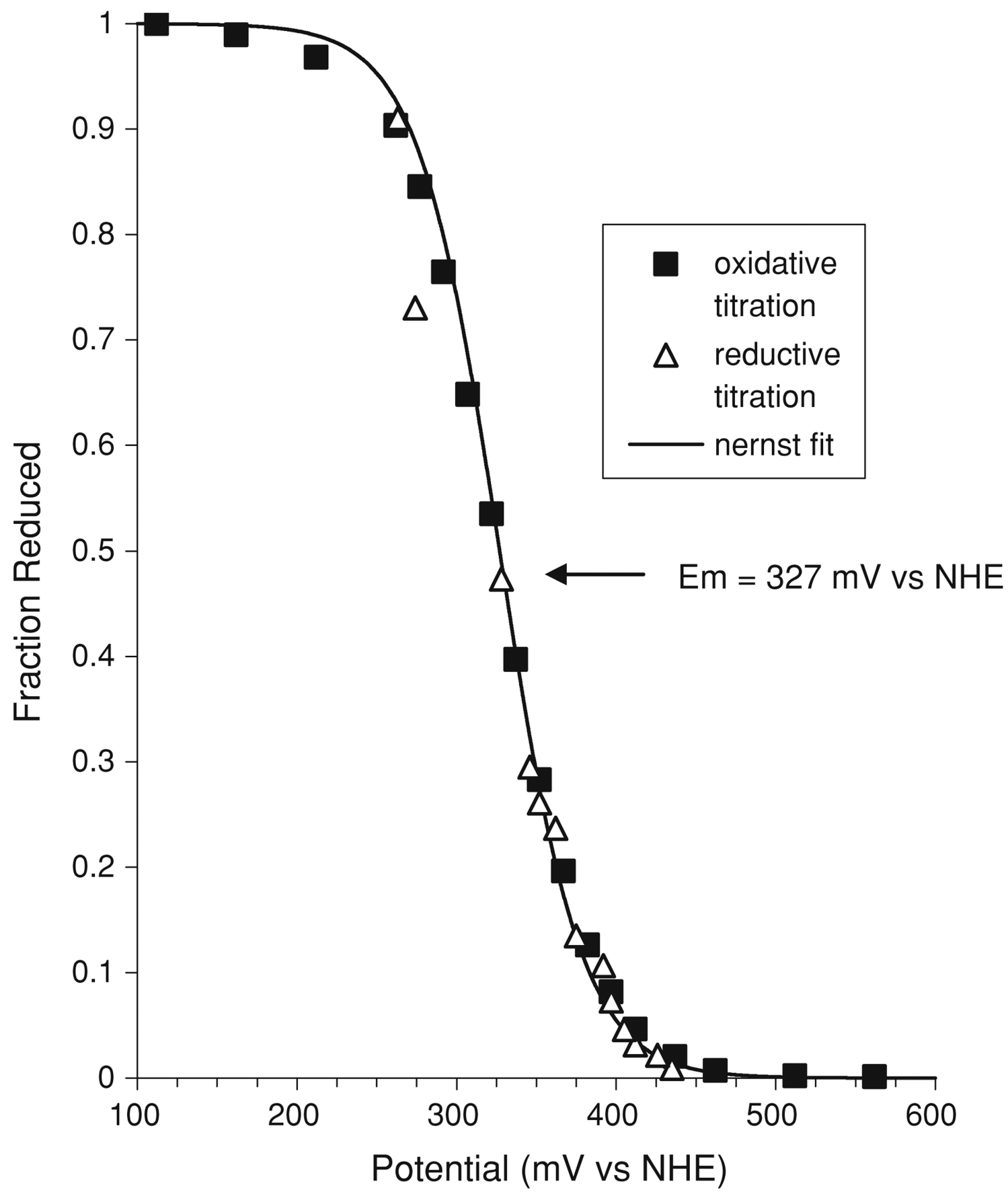

Fig. 7.

Redox titration of purifed cyt $c_{6}$ from A. marina oxidative titration (black squares), reductive titration (white triangles), and Nernst fit (straight line) 